\title{
Spontaneous nucleation and dynamics of kink defects in zigzag arrays of trapped ions
}

\author{
S. Ejtemaee and P. C. Haljan \\ Department of Physics, Simon Fraser University, Burnaby, BC, V5A 1S6, Canada
}

(Dated: February 11, 2013)

\begin{abstract}
The spontaneous nucleation and dynamics of topological kink defects have been studied in trapped arrays of 41-43 $\mathrm{Yb}$ ions. The number of kinks formed as a function of quench rate across the linear-zigzag transition is measured in the under-damped regime of the inhomogeneous Kibble-Zurek theory. The experimental results agree well with molecular dynamics simulations, which show how losses mask the intrinsic nucleation rate. Simulations indicate that doubling the ion number and optimization of laser cooling can help reduce the effect of losses. A range of kink dynamics is observed including configural change, motion and lifetime, and behavioral sensitivity to ion number.
\end{abstract}

PACS numbers: 37.10.Rs, 52.27.Jt, 64.60.an, 64.60.Q-

The nucleation of topological defects during a symmetry-breaking phase transition has many physical realizations. For continuous phase transitions, the Kibble-Zurek mechanism (KZM) provides an intuitive model of domain and defect formation [1, 2] and predicts a power-law scaling for the number of defects formed as a function of transition quench rate [2 -4$]$. Several experimental tests of the KZM have been done with different degrees of success [5 21], all with the goal of examining universal behavior across diverse physical systems.

Following a recent proposal [22], we report on the nucleation and dynamics of topological defects in arrays of trapped ions, following a quench of the linear-zigzag transition. Such studies are well suited to this experimental system, since it is simple enough to make direct comparison to theory, highly controllable, and efficient in that the defects are imaged in situ and the trapped sample can be recycled repeatedly across the transition.

Laser-cooled ions held in a linear Paul trap [23] with strong transverse confinement organize into a onedimensional (1D) linear crystal with an inhomogeneous axial density [Fig. 1(a)]. If the transverse confinement is relaxed slowly, the linear ion crystal undergoes a continuous, structural phase transition to a $2 \mathrm{D}$ zigzag configuration, beginning at the center of the crystal 23 28]. Two broken-symmetry states are possible [Fig. 1(b) or (c)]. Rapidly quenching the transverse confinement across the transition can lead to crystal structures containing spontaneously nucleated topological defects (kinks), which are formed by the interface between domains of opposite symmetry [Figs. 1(d)-(g)] 22, 29, 30]. Del Campo et al. 22, 31 have applied the KZM formalism to the nonequilibrium dynamics of the quench and calculated the number of kinks formed, which depends on the quench rate and the damping rate $(\eta)$ of the system, controlled by Doppler laser cooling. In the under-damped regime, where the system's response time is dominated by the inertial dynamics of the soft transverse zigzag mode [4, 28], the rate of defect formation is independent of $\eta$ and given by a power law, $\left(2 \delta_{0} / 2 \tau_{Q}\right)^{b}$, where $2 \tau_{Q}$ is the total quench time, and $2 \delta_{0}$ defines the magnitude of the

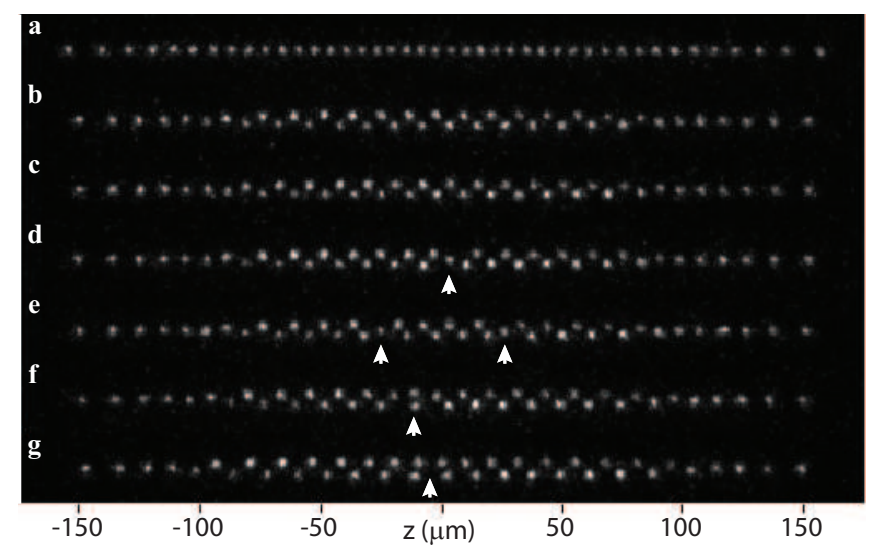

FIG. 1. Images of crystals of $42{ }^{174} \mathrm{Yb}$ ions in various configurations: (a) 1D linear crystal, (b) "zig", (c) "zag", (d) zigzag with a single discrete kink (indicated by arrow), (e) two discrete kinks (kink and anti-kink), and (f)-(g) single extended kinks. The axial trap frequency for all images is $\omega_{z} / 2 \pi=37.6$ $\mathrm{kHz}$, while the weak transverse frequency, $\omega_{y} / 2 \pi$, is (a) 658 kHz, (b)-(d) $414 \mathrm{kHz}$, (e) $380 \mathrm{kHz}$ and (f) $342 \mathrm{kHz}$. The corresponding quench end-points, $V_{f}$, are (b)-(d) $4.8 \mathrm{~V}$, (e) $5.3 \mathrm{~V}$, and (f) $5.8 \mathrm{~V}$. The imaging system looks at $45^{\circ}$ to the transverse $y$-axis where the zigzag displacement occurs.

quench, which is linear in terms of quadratic transverse trap frequency. The power-law scaling is affected by the system inhomogeneity [22, 32], an effect which is largely unexplored experimentally. Due to the inhomogeneous density, the propagation speed of the transition front decreases from the crystal center to the edges. This limits the size of the region where non-adiabatic dynamics, and so nucleation, is possible, and for the under-damped regime $\left(\eta^{3} \ll \delta_{0} / \tau_{Q}\right)$, gives a power-law scaling of $b=4 / 3$ (IKZM regime) 22]. Following calculations in other contexts [15, 34, 35], it has been pointed out that for small numbers of ions or slow quenches, where the size of the nucleation region is smaller than the correlation length, the scaling doubles to $b=8 / 3$ (DIKZM regime) [36].

Simultaneous experiments to ours have recently reported kink nucleation in ion arrays due to a temperature quench of a plasma cloud [37], and a measurement 
of the DIKZM power law [36]. Compared to the latter results, our measurements of nucleation are also due to a quench across the structural transition but use a different experimental approach, including more ions and a shallower quench into the zigzag phase so that kinks retain their localized, discrete form. Moreover, we explore postnucleation dynamics of discrete kinks including transformation of shape, motion and lifetime in the crystal, and sensitivity of behavior to unit change in ion number.

The experimental set-up is similar to that previously reported [38, 39]. We use a linear Paul trap, operating at a radio frequency (rf) of $\Omega_{r f} / 2 \pi=12 \mathrm{MHz}$, and composed of four rods with an ion-to-rod spacing of $0.7 \mathrm{~mm}$ and two end-caps separated by $2.5 \mathrm{~mm}$, to form a nominally harmonic confining potential for ions. Photo-ionization is used to load a pure crystal of $42-43^{174} \mathrm{Yb}$ ions 39, 40], which arrange as a linear string in a trap with an axial secular frequency of $\omega_{z} / 2 \pi=37.6 \mathrm{kHz}$ and transverse frequencies of $\omega_{x} / 2 \pi=679 \mathrm{kHz}$ and $\omega_{y} / 2 \pi=658 \mathrm{kHz}$ [Fig. 1(a)]. A single laser beam at $369.5 \mathrm{~nm}$ (with axial FWHM of $280 \mu \mathrm{m}$ ) is used for fluorescence imaging of the ions and for Doppler cooling along all principal axes of the trap. The 369.5-nm laser is linearly polarized at $6^{\circ}$ with respect to a 5.9-G magnetic field. A relatively large laser detuning of $-33 \mathrm{MHz}$ (1.5 linewidths) is used to suppress the effects of micromotion on fluorescence and cooling [41], arising from a non-zero component of the trap rf electric field along the axial direction (up to 2500 $\mathrm{V} / \mathrm{m}$ at the edges of a 43-ion crystal). With an effective saturation parameter of $s_{0}=2.7$, the estimated cooling rate is uniform to a factor of 2 or better across the crystal with variation primarily at the edges, and the calculated cooling time, $\eta^{-1}$, at the center of the crystal is $200-400$ $\mu \mathrm{s}$ 42]. The cooling rate places the experiment deeply in the under-damped regime for quench rates considered.

To quench the system through the linear-zigzag transition, we apply a transverse dc quadrupolar potential using the trap rods. The secular frequency along the transverse $y$-axis, obtained from single-ion calibrations, decreases with the applied dc voltage, $V(\geq 0)$, according to $\omega_{y}(t)=\omega_{y 0} \sqrt{1-V / V_{0}}$ where $\omega_{y 0} / 2 \pi=658(1) \mathrm{kHz}$ and $V_{0}=7.95(2) \mathrm{V}$ with errors including drift. The $x$-axis frequency increases in an equivalent way. The quench waveform is linear $\left(V(t)=V_{f} \cdot t /\left(2 \tau_{Q}\right)\right)$ with a quench end-point of $V_{f}=4.2-5.8 \mathrm{~V}$, a ramp time of $2 \tau_{Q}=10-70$ $\mu \mathrm{s}$, and a maximum distortion of $50 \mathrm{mV}_{p k}$ after filtering. The quench also incorporates small adjustments to the end-caps and rods to maintain a constant axial secular frequency and to maintain the center of the crystal at the rf null in all dimensions. The linear form of our quench in terms of $\omega_{y}^{2}$ is as assumed in the KZM theory [22], with the one modification that we use an asymmetric ramp across the critical point. For example, in a 42-ion string with $\omega_{z} / 2 \pi=37.6 \mathrm{kHz}$, where the critical transverse frequency at the center of the crystal is $\omega_{y}^{c}(0) / 2 \pi=619(3)$ $\mathrm{kHz}$ (or equivalently, 0.92(6) V) [43], our quench starts at
$658 \mathrm{kHz}\left(V_{i}=0.0 \mathrm{~V}\right)$, which ensures a sufficiently linear configuration [44], and continues further past the critical point, for example to $414 \mathrm{kHz}\left(V_{f}=4.8 \mathrm{~V}\right)$ to involve about half of the crystal in the zigzag phase.

Each experimental run begins with $53 \mathrm{~ms}$ of cooling in the linear configuration, followed by the quench (with cooling always active). Images of the initial linear and subsequent zigzag configurations are recorded with an exposure time of $3 \mathrm{~ms}$. A $200-\mu \mathrm{s}$ ramp recycles the crystal to the linear structure. Data is acquired in batches of 100-700 runs over 3 minutes. Our data sets contain $80 \%$ of runs approximately equally divided between 42 and 43 ions with 41 or fewer ions in the remainder. Occasionally, the fluorescence of an ion is interrupted by internal-state pumping or molecule formation through background-gas collisions [39]. Runs with dark ions within the zigzag region of the crystal ( $\leq 14 \%$ of runs, typical) are excluded. Image analysis is used to identify locations of any kinks in the zigzag structure. For configurations without kinks, temporal statistics of the two symmetry-broken phases are nominally random with a bias of $\lesssim 1 \%$ and a normalized binary autocorrelation on adjacent runs of $\lesssim 1 \%$.

In the first experiment, we explore the formation of two types of kink 29]: discrete kinks, which are localized to about 3 ion-sites [Figs. 1(d),(e)], and extended ones, which involve more sites and occur in crystals brought far into the zigzag region [Figs. 1(f), (g)]. Figures 2(a)(c) show the observed axial distribution of both types and their total numbers as a function of the quench end-point, $V_{f}$. The data, for $41-43$ ions, is taken at a constant ramp rate of $0.19 \mathrm{~V} / \mu$ s so that the nucleation dynamics near the critical point is expected to be same. Any differences are due to post-nucleation dynamics during the remainder of the ramp through to detection. To understand the outcome of the experiment, we have performed molecular dynamics simulations with no free parameters: The ion trap is treated as an ideal harmonic potential limited to two dimensions. The small axial anharmonicity in the experiment is modeled in an effective way by shifting the axial frequency from 37.6 to $38.2 \mathrm{kHz}$ [43]. The effect of the Doppler cooling laser is modeled explicitly [45] and includes the steady-state fluorescence behavior of ${ }^{174} \mathrm{Yb}^{+}$and all laser calibrations excluding beam profile [38]. Simulations for 42 ions reveal similar distributions to Figs. 2(a) and (b). There is also a good qualitative match in the numbers of discrete and extended kinks between simulation and experiment [see Fig. 2(c)]. From the simulations, we find that discrete kinks are nucleated in the central 5-10 sites of the crystal. Then, there is an outward motion and loss of the discrete kinks, propelled by the gradient in zigzag amplitude and axial density, which affect the underlying potential for the kinks. As the quench end-point increases towards $5.0 \mathrm{~V}$, the number of discrete kinks and the width of their distribution increase since more of the dispersing kinks are trapped in a deeper and wider zigzag region. Above $5.0 \mathrm{~V}$, the 

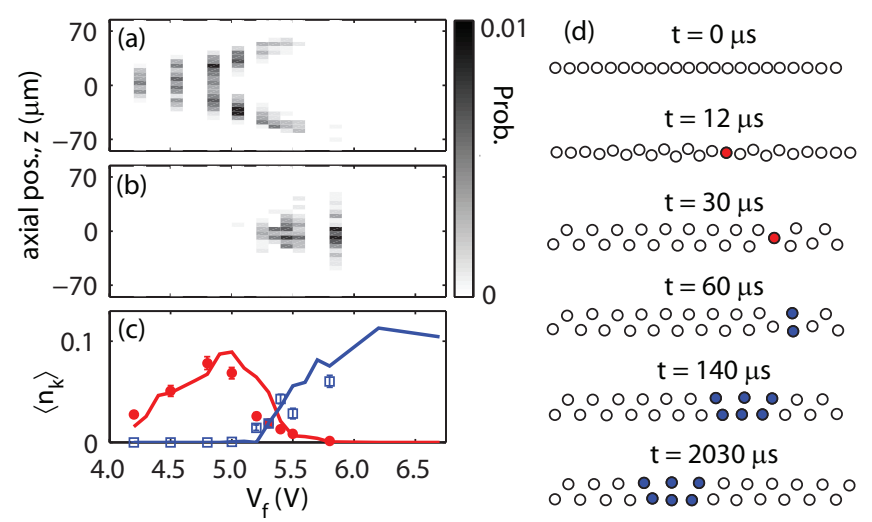

FIG. 2. (color online) Observed axial position distribution of (a) discrete and (b) extended kinks versus quench end-point. All data taken at a quench rate of $0.19 \mathrm{~V} / \mu \mathrm{s}$. (c) Average number per run of discrete kinks (red circles) and extended kinks (blue squares) for distributions in (a) and (b). Data points, for 41-43 ions, include 1000-2200 runs. Errors calculated assuming binomial statistics. Lines are a simulation for 42 ions with no free parameters, and shown $2 \mathrm{~ms}$ after the quench, $2 / 3$ of the camera exposure time. Statistical uncertainties in simulation comparable to experiment ones shown. (d) Simulation frames showing evolution after nucleation of a discrete kink (red) into an extended kink (blue) at $V_{f}=5.8 \mathrm{~V}$ $\left(\omega_{y} / 2 \pi=342 \mathrm{kHz}\right)$. Middle $144 \mu \mathrm{m}$ of a 42 -ion crystal shown.

number of discrete kinks collapses when, as seen in the simulation, those at the center of the crystal distort to extended form. Extended kinks can also migrate to the center [Fig. 2(d)], suggesting a change in the underlying potential for the kinks and leading to the well-centered distribution observed in the experiment [Fig. 2(b)]. Due to reduced losses, the number of extended kinks in the simulation saturates at a value 1.4 times higher than the peak in discrete number. The experiment data ends at $5.8 \mathrm{~V}$, well before the $\sim 7.1-\mathrm{V}$ onset of another structural transition to a different $2 \mathrm{D}$ configuration. As this transition is approached, we see an enhancement in (otherwise negligible) background kink nucleation, due to energy transfer events such as background-gas collisions.

Next, we proceed to study kink nucleation as a function of quench rate. With an eye towards subsequent dynamics studies, we work with discrete kinks and operate at $V_{f}=4.8 \mathrm{~V}$, where their maximum number is detected [Fig. 2(c)]. Figure 3(a) shows a log-log plot of the average number of kinks detected per run (black triangles) as a function of scaled rate, $1 /\left(\omega_{z} \cdot 2 \tau_{Q}\right)$. Data for $41-43$ ions is combined. As the quench rate increases from $1 /\left(\omega_{z} \cdot 70 \mu \mathrm{s}\right)$, the number of kinks grows, peaks at $1 /\left(\omega_{z} \cdot 15 \mu \mathrm{s}\right)$ and begins to drop. Figure 3(a) also includes results of molecular dynamics simulations, performed as described above. The first simulation (solid black line) shows the number of kinks $2 \mathrm{~ms}$ after the quench $(2 / 3$ of the exposure time in the experiment), by which time the kink number has stabilized. The simu-

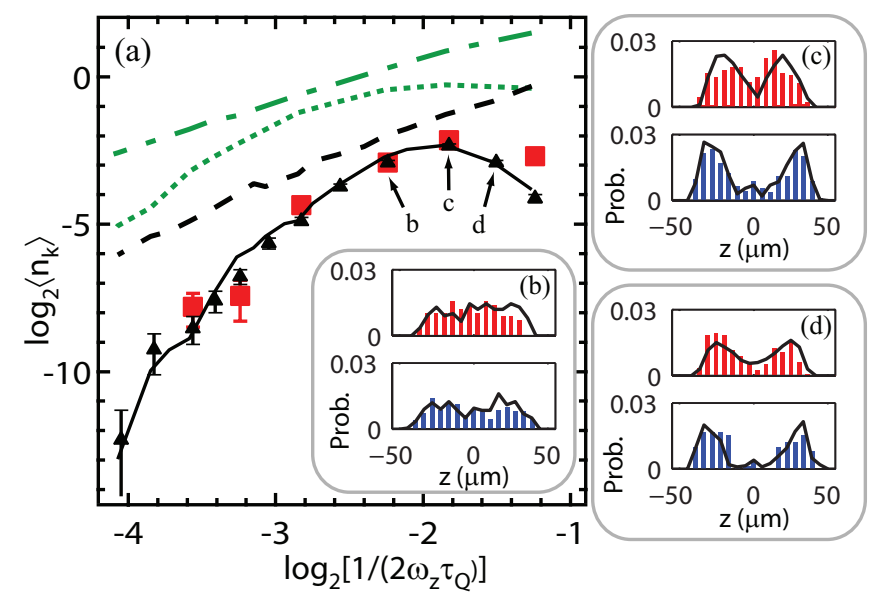

FIG. 3. (color online) (a)Average number of kinks observed per run vs. scaled quench rate for standard trap with $\omega_{z} / 2 \pi=$ $37.6 \mathrm{kHz}$ (triangles), and for one with secular frequencies reduced by 2 (red squares) and quench changed accordingly. Data, for 41-43 ions, include 1000-5000 runs. Error bars are calculated binomial, approximately consistent with fluctuations. Solid line is a molecular dynamics simulation with parameters including $\omega_{z} / 2 \pi=38.2 \mathrm{kHz}$ (see text), and shown $2 \mathrm{~ms}$ after the quench. Statistical uncertainties of simulation less than or equal to the experiment. Dashed black line is the same simulation with kinks counted shortly after nucleation ( $\geq 7 \mu \mathrm{s})$. To suppress thermal fluctuations, the kink search is limited to the zigzag region with minimum amplitude of 0.54 $\mu \mathrm{m}$. Dotted and dash-dotted lines (green) show kink number $2 \mathrm{~ms}$ after quench and shortly after nucleation, respectively, for a simulation of 84 ions with optimized Doppler cooling (detuning $-11.3 \mathrm{MHz}, s_{0}=2.0$ ) and with $\omega_{z} / 2 \pi=20.6 \mathrm{kHz}$, chosen to match the transverse critical frequency for 42 ions at crystal center. Simulation plot scaled using $\omega_{z} / 2 \pi=38.2$ $\mathrm{kHz}$ for direct comparison to 42 ions. (b)-(d) Observed position distributions of kinks for 42 ions (red, upper) and 43 (blue, lower) in standard trap at quench time $2 \tau_{q}$ of (b) 20 , (c) 15 and (d) $12 \mu \mathrm{s}$ with simulations (lines) for comparison.

lation matches the observed number of kinks extremely well, and reproduces features in the observed kink distributions, related to rapid, post-nucleation dynamics [Fig. 3(b)-(d)]. To understand the effect of kink losses before detection in the experiment, we use the simulation to assess the "early-counted" number of kinks shortly after nucleation (dashed black line). At fast quenches, there is enhanced loss due to kinetic energy from nonadiabatic excitation of vibrational modes, and before the crystal is re-cooled. Kink pair annihilation also plays a minor role. At slow quenches, we again see a loss of kinks, which worsens with decreasing quench rate. In this case, the zigzag region expands too slowly to trap the kinks, and they escape to the zigzag edges during the quench.

Although kink losses in the experiment mask the intrinsic nucleation rate - see, however, Ref. [36] - we comment briefly on the power law scaling for the simulation and experiment. Figure 3(a) covers about three octaves in quench rate separated by $\log _{2}\left(1 / 2 \omega_{z} \tau_{Q}\right)=-3.1$ and 
-2.1 . The KZ theory [22, 36] suggests that our data covers two regimes of nucleation: For the slow-quench range, we expect a DIKZM power law of $8 / 3=2.67$, which agrees with the fit value of 2.5(2) for the early-counted simulation (with significant systematic error associated with rapid losses). For the fast-quench range, where the nucleation region is large enough that nucleation of two or more kinks becomes significant in the simulation, one expects to cross over towards the IKZM power law of $4 / 3=1.33$. The early-counted simulation gives a fit value of 1.7(1). We note that all cases of two kinks observed in the experiment [Fig. 1(d)], albeit rare, occur in or adjacent to the fast-quench region. Finally, in the middle octave of quench rate, where the kink losses are least, a fit to the experimental data gives a power law of 3.3(2), compared to the early-counted simulation value of 2.0(1).

For comparison, we also measure kink nucleation in a trap with all secular frequencies reduced by a factor of 2 (red squares in Fig.3(a)). Overall, the data match results at higher trap frequency, which shows the weak sensitivity of nucleation and losses to effective cooling rate, $\eta / \omega_{z}$, over such a variation, except perhaps for losses at the fastest quenches [46]. Of several possibilities to reduce the losses in our experiment, we note that an increase in ion number can help, particularly for slow quenches by reducing the crystal inhomogenieties that transport discrete kinks outward. As shown in Fig. 3(a), a doubling of ion number (and using twice better cooling) already leads to significant reduction in losses over the same quench range, albeit in a scaling regime closer to IKZM.

We conclude with an investigation of the dynamics of single discrete kinks, and measure their axial motion in the crystal and their lifetime. The experiments are performed at $V_{f}=4.8 \mathrm{~V}$ and $2 \tau_{Q}=20 \mu \mathrm{s}$, for which the initial distribution of the kinks is relatively uniform [Fig. 3(b)]. Figures 4(a) and (b) show the trajectories of kinks in crystals of 42 and 43 ions for three initial axial positions. The kinks move between sites corresponding to local potential wells separated by the axial lattice spacing $(5.6 \mu \mathrm{m})$ [30]. The $40-\mathrm{ms}$ frame rate of our camera does not permit us to resolve fast dynamics, including the motion between sites. Nevertheless, it can be seen that, for 42 ions, most of the kinks that begin at the center of the crystal either stay there or move by one site. In comparison, the central kinks in a string of 43 ions migrate to the edges faster. For both cases, kinks closer to the edges tend to move outwards, as expected.

In Figs. 4(c) and (d), we show the exponential lifetime of kinks as a function of their initial position as well as the distributions of kink positions at $t=0 \mathrm{~s}$ and $t=0.4 \mathrm{~s}$. In Fig. 4(c), we can see that the lifetime of kinks for 42 ions is highest near the middle of the crystal (with the $\sim 2$-s value likely limited by background-gas collisions), and decreases roughly symmetrically towards the edges. Losses due to the position-dependent lifetime largely account for the observed time evolution of the po-
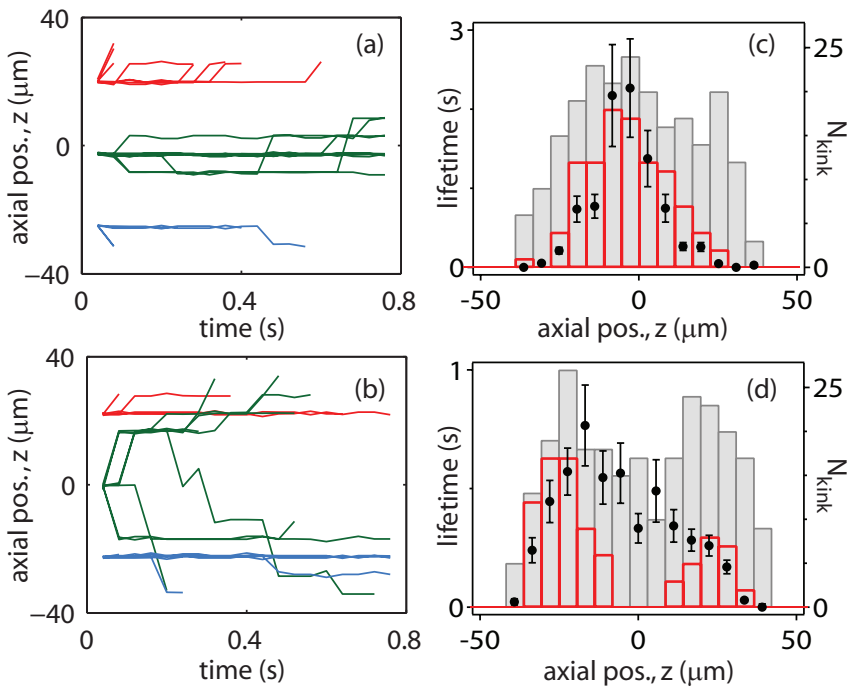

FIG. 4. (color online) (a)-(b) Axial trajectories of discrete kinks in zigzag crystals $\left(V_{f}=4.8 \mathrm{~V}\right.$ ) with (a) 42 and (b) 43 ions for three different initial positions: center and four sites away on both sides. The number of trajectories for each case is 11 with fewer for $z<0$. (c)-(d) Exponential lifetime of kinks (filled circles) vs initial position in crystals of (c) 42 and (d) 43 ions. Lifetimes and their uncertainties obtained from a maximum likelihood estimate including binning due to camera frame rate. Also shown are axial position distributions at $t=0 \mathrm{~ms}$ (filled gray bars) and $t=400 \mathrm{~ms}$ (open red bars).

sition distribution. In Fig. 4 (d), for 43 ions, the $0.3-0.5 \mathrm{~s}$ lifetime of central kinks contrasts with their complete absence in the position distribution after $0.4 \mathrm{~s}$, and points to their migration away from the deeper zigzag region at the center of the crystal [47]. Simulations show that this is associated with new dynamics in which the kink hops two sites (as observed in Fig. 4(b) center) via a transient extended-like form. The difference between 42 and 43 ions is understandable, considering that the experiment is performed at $V_{f}=4.8 \mathrm{~V}$, near to the sharp onset of the bimodal distribution in Fig. 2(a). Since the zigzag phase begins $0.3 \mathrm{~V}$ lower for 43 ions, the onset of the bimodal distribution will also occur at a lower voltage.

To conclude, we have measured the number of kinks formed in ion crystals as a function of quench rate in the under-damped regime of the KZM, and have shown how losses mask the intrinsic nucleation rate. Although simultaneous results based on a deeper quench and extended kinks show lower loss [36], our method of nucleation assessment should be advantageous for larger ion numbers with multiple kinks, since the localized character of the discrete kinks will help reduce interaction-dependent losses. We have also studied the post-nucleation dynamics of kinks, and have observed long lifetimes, which may allow future explorations of internal kink dynamics [29, 30]. A natural future direction is the study of linear-zigzag dynamics in the quantum regime [48]. 
The authors thank Malcolm Kennett and Jeff McGuirk for helpful comments on the manuscript. This work is supported by NSERC and the CFI LOF Program and has been enabled by computing resources provided by WestGrid and Compute/Caleul-Ganta.

[1] T. W. B. Kibble, Journal of Physics A, 9, 1387 (1976)

[2] W. H. Zurek, Nature, 317, 505 (1985).

[3] P. Laguna and W. H. Zurek, Phys Rev. Lett., 78, 2519 $(1997)$.

[4] P. Laguna and W. H. Zurek, Phys. Rev. D, 58, 085021 (1998)

[5] For a partial review, see: T. Kibble, Physics Today, 60, 47 (2007).

[6] I. Chuang, R. Durrer, N. Turok, and B. Yurke, 251, 1336 (1991).

[7] M. J. Bowick, L. Chandar, E. A. Schiff, and A. M. Srivastava, 205, $949(1994)$.

[8] P. C. Hendry, N. S. Lawson, R. A. M. Lee, P. V. E. Mcelintock, and C. D. H. Williants, Nature, 368, 315 (1994).

[9] M. E. Dodd, P. C. Hendry, N. S. tawson, P. V. E. McClintock, and C. D. H. Williams, Phys. Rev. Lett., 81, 3703 (1998).

[10] V. M. H. Ruutu, V. B. Eltsov, A. J. Gill, M. Kibble, T. W. B.and Krusius, Y. G. Makhlin, B. Placais, G. E. Volovik, and W. Xu, Nature, 382, 334 (1996).

[11] C. Bauerle, Y. M. Bunkov, S. N. Fi hher, H. Godfrin, and G. R. Pickett, Nature, 382, 332 (1996).

[12] S. Ducci, P. L. Ramazza, W. Gonzdlez-Viñas, and F. T. Arecchi, Phys. Rev. Lett., 83, 5210 (1999).

[13] A. Maniv, E. Polturak, and G. Koren, Phys. Rev. Lett. 91, 197001 (2003).

[14] R. Monaco, J. Mygind, M. Aaroe, H. J. Kivers, and V. P. Koshetets, Phys. Rev. Lett., 90, 100004 (2000);

[15] R. Monaco, J. Mygind, R. J. Rivers and V. P. Koshelets, Phys. Rev. B, 80, 180501 (2009).

[16] L. E. Sadler, J. M. Higbie, S. R. Leslie, M. Vengalattore, and D. M. Stamper-Kurn, Nature, 443, 312 (2006).

[17] D. R. Scherer, C. N. Weiler, T. N. Neely, and B. P. Anderson, Phys. Rev. Lett., 98, 110402 (2007).

[18] C. N. Weiler, T. W. Neely, D. R. Scherer, A. S. Bradley, M. J. Davis, and B. P. Anderson, Nature, 455, 948 (2008).

[19] D. Chen, M. White, C. Borries, and B. DeMarco, Phys. Rev. Lett., 106, 235304 (2011).

[20] S. C. Chae, N. Lee, Y. Horibe, M. Tanimura, S. Mori, B. Gao, S. Carr, and S.-W. Cheong, Phys. Rev. Lett. 108, 167603 (2012)

[21] S. M. Gritin, M. Lilienblum, K. Lelaney, Y. Kumagal, M. Fiebig and N. A. Spaldin, arXiy:1204.3785.

[22] A. del Campo, G. De Chiara, G. Morigi, M. B. Plenio, and A. Retzker, Phys. Rev. Lett., 105, 075701 (2010).

[23] M. G. Raizen, J. M. Gilligan, J. C. Bergquist, W. M. Itano, and D. J. Wineland, Phys. Rev. A, 45, 6493 (1992).

[24] J. P. Schiffer, Phys. Rev. Lett., 70 818 (1993)

[25] D. H. E. Dubin, Phys. Rev. Lett., 71, 2753 (1993).

[26] D. H. E. Dubin and T. M. O'Neil, Rev. Mod. Phys., 71,

\section{7 (1999)}

[27] D. G. Enker, M. M. Schauer, J. J. Gomez, M. S. Gulley, M. H. Holzscheiter, P. G. Kwiat, S. K. Lamoreaux, C. G. Peterson, V. D. Sandberg, D. Tupa, A. G. White, R. J. Hughes, and D. F. V. James, Phys. Rev. Lett., 85, 2466 (2000).

[28] S. Fishman, G. De Chiara, T. Calarco, and G. Morigi, Phys. Ret. B, 77, 064111 (2008)

[29] H. Landa, S. Marcovitch, A. Retzker, M. B. Plenio, and B. Reznil, Phys. Rev. Lett., 104, 043004 (2010).

[30] O. M. Braun and Y. S. Kivshar, The Frenkel-Kontorova Model: Concepts, Methods, and Applications (Springer, 2009).

[31] G. D. Chiara, A. del Campo, G. Morigi, NI. B. Plenio, and A. Retzker, New Jounnal of Physics, 12, 115003 (2010).

[32] W. H. Z4rek, Phys. Rev. Lett., 102, 105702 (2009); A. del Campo, A. Retzker, and M. B. Plenio, New Journal of Physics, 13, 083022 (2011).

[33] J. Dziarnaga, M. Tylutki, and W. H. Zurek, Phys. Rev. $\mathrm{B}, \mathbf{8 6}, 144521(2012)$.

34 H. Saito, Y. Kawaguchi, and M. Ueda, Phys. Rev. A, 76, $043613(2007)$.

\begin{tabular}{l|l|l|}
35 & J. Dziarnaga, J. Meisner, and W. H. Zurek, Phys. Rev.
\end{tabular} Lett., 101, 115701 (2008)

[36] K. Pyka J. Keller, H. L. Partner, R. Nigmatullin, T. Burge meister, D. M. Meier, K. Kuhlmann, A. Retzker, M. \$. Plenio, W. H. Zurek, A. d. Campo, and T. E. Mehlstäupler, arXiv:1211.7005.

[37] M. Mielepz, H. Landa, J. Brox, S. Kahra, G. Leschhorn, M. Alber, B. Reznik, and T. Schaetz, arXiv:1211.6867.

[38] S. Ejtemqee, R. Thomas, and P. C. Haljan, Phys. Rev. A, 82, 063419 (2010).

[39] J. Liang and P. C. Haljan, Phys. Rev. A, 83, 063401 (2011).

[40] C. Balzer, A. Braun, T. Hannemann, C. Paape, M. Ettler, W. Neuhauser, and C. Wunderlich, Phys. Rev. A, 73, 041407 (2006).

[41] D. J. Betkeland, J. D. Miller, J. C. Bergquist, W. M. Itano, and D. J. Wineland, J. Appl. Phys., 83, 5025 (1998).

[42] W. M. It ano and D. J. Wineland, Phys. Rev. A, 25, 35 (1982).

[43] The observed value of $\omega_{y}^{c}(0) / 2 \pi$ is slightly higher than expected (1 percent), roughly consistent with measured axial anh armonicity vs. ion number, and is accounted for in simulations by a shift in $\omega_{z}$.

[44] The soft-mode frequency at quench start is also larger than the KZM freeze-out value. Simulations of quenches starting at higher transverse frequency $(883 \mathrm{kHz})$ give quantitatively similar kink numbers to those observed.

[45] R. Casdorff and R. Blatt, Applied Physics B, 45, 175 (1988)

[46] The overall weak sensitivity shown also supports ignoring small (spatial) variations in cooling, corroborated by good agrement with simulations neglecting them. Note the cooling beam is also larger for this experiment.

[47] Asymmet ries in the lifetime distributions are likely due to experimental imperfections. Longer lifetime of outer kinks for 43 vs. 42 ions and changes in dynamics with ion number and density are interesting and will be explored in a future work.

[48] A. Retzker, R. C. Thompson, D. M. Segal, and M. B. Plenio, Phys. Rev. Lett., 101, 260504 (2008). 